\title{
Bulked segregant analysis for relative water content to detect quantitative trait loci in wheat under drought stress
}

\author{
M.R. Naroui Rad ${ }^{1,2}$, M. Abdul Kadir ${ }^{1}$, M.Y. Rafii ${ }^{3}$, H.Z.E. Jaafar ${ }^{4}$ and \\ M.R. Naghavi ${ }^{5}$
}

${ }^{1}$ Agrotechnology Department, Faculty of Agriculture, University Putra Malaysia, Serdang, Selangor, Malaysia

${ }^{2}$ Agriculture and Natural Resources Research Center, Sitan, Iran

${ }^{3}$ Institute of Tropical Agriculture, University Putra Malaysia, Serdang,

Selangor, Malaysia

${ }^{4}$ Crop Science Department, Faculty of Agriculture, University Putra Malaysia, Serdang, Selangor, Malaysia

${ }^{5}$ Department of Agronomy and Plant Breeding,

Faculty of Agriculture, University of Tehran, Karaj, Iran

Corresponding author: M.R. Naroui Rad

E-mail: narouirad@gmail.com

Genet. Mol. Res. 11 (4): 3882-3888 (2012)

Received February 7, 2012

Accepted July 3, 2012

Published November 12, 2012

DOI http://dx.doi.org/10.4238/2012.November.12.5

ABSTRACT. This study was carried out to evaluate the genetic effect of quantitative trait loci (QTLs) conferring drought tolerance in wheat. A population of $120 \mathrm{~F}_{2}$ individuals from the cross between the droughttolerant S-78-11 and drought-sensitive Tajan cultivars were analyzed for their segregation under drought stress conditions. The relative water content under drought stress conditions exhibited continuous variation, indicating the minor gene effects on the trait. Single-marker analysis (SMA) was carried out to detect the main QTL association with drought tolerance. The SMA results revealed that the simple sequence repeat markers GWM182 and GWM292 on chromosome 5D and GWM410 on 
chromosome 5 A exhibited significant association with drought tolerance, accounting for 30,22 , and $21 \%$ of the total variation, respectively. The 3 genetic loci, especially GWM182, can be used in marker-assisted selection methods in drought tolerance breeding in wheat.

Key words: Drought tolerance; Bulked segregant analysis; QTL; SSR markers; Wheat

\section{INTRODUCTION}

A lack of water is among the most common abiotic stresses, limiting wheat distribution and productivity worldwide. Drought tolerance improvement is probably one of the most difficult tasks for cereal farmers to accomplish. In nature, the vast majority of physiological traits are quantitative; quantitative genetics can clarify the characteristics of continuous phenotypic distribution and help estimate the number of loci affecting a character, average gene action, and the value of interaction between quantitative trait loci (QTLs) and the environment (Tanksley, 1993).

The difficulty stems from the diversity and unpredictability of drought conditions in the field, and from the diversity of drought tolerance strategies developed by plants that are targeted and subjected to selection criteria. However, substantial progress has been made during recent years on different plant species in terms of the physiology, genetics, and molecular biology of drought tolerance. The traits in cereals for which a positive relationship with yield has been demonstrated are osmotic adjustment (Blum, 1989), water-use efficiency measured through carbon isotope discrimination (Farquhar and Richards, 1984), and relative water content (RWC) (Schonfeld et al., 1988; Peltonen-Sainio and Mäkelä, 1995; Merah, 2001). Traits allowing the evaluation of plant water status, particularly RWC, could provide an idea of the water deficit level in a plant at a specific time point. As RWC is related to cell volume, its measurement on the flag leaf may closely reflect the balance between water supply to the leaf and transpiration rate (Sinclair and Ludlow, 1985). The advent of molecular markers has enabled the dissection of complex traits such as drought resistance via QTL analysis.

Most previous mapping studies focused on QTLs responsible for improved productivity under arid conditions (Tuberosa and Salvi, 2006). The use of molecular markers as selection criteria would also represent an important surrogate for physiological trait measurements (Price and Courtois, 1999). Curiously, studies reporting molecular markers associated with plant water status-related traits in cereals are scarce. However, several QTLs were reported for the barley-segregating population issued from the cross between Tadmor and Er/Apm (Teulat et al., 1998). Moreover, the latter study was conducted in controlled conditions and at a young stage of growth. The aim of this study was the detection of QTLs for the RWC in wheat by bulked segregant analysis (BSA) and simple sequence repeat (SSR) primer pairs.

\section{MATERIAL AND METHODS}

\section{Plant materials and measurement}

Individual wheat $\mathrm{F}_{2}$ plants from a cross between the water stress-tolerant S-78-11 and water stress-sensitive Tajan cultivars were used to prepare the water-tolerant and water-sensitive 
bulks. Seeds were sown in pots with a soil mixture containing soil, sand, and organic matter in a 1:1:1 ratio. The pots were irrigated after $75 \%$ depletion of the soil water to apply drought stress.

Each pot was filled with $3 \mathrm{~kg}$ air-dried soil, and soil field capacity was calculated based on the soil dry weight. Water stress treatments were $75 \%$ moisture depletion of field capacity by weight. The pots were weighed at 2-day intervals to compensate the water loss by evapotranspiration, and irrigation was performed after $75 \%$ depletion of soil field capacity. RWC was measured at the heading stage for all genotypes.

Three different flag leaves were randomly sampled and recorded. The fresh weight (FW) was immediately recorded after leaf excision. The leaves were incubated in distilled water for $24 \mathrm{~h}$ at $4^{\circ} \mathrm{C}$ in darkness and the turgid weight (TW) was recorded. The dry weight (DW) was then measured after $48 \mathrm{~h}$ at $80^{\circ} \mathrm{C}$. The RWC was calculated according to Barrs and Watherley (1962) as RWC (\%) = [(FW - DW) / (TW - DW) ] x 100.

\section{DNA isolation}

Genomic DNA was extracted from $50 \mathrm{mg}$ leaf tissue by using the Qiagen DNeasy Plant Mini Kit (Germany) according to manufacturer instructions, except that a pestle and mortar was used instead of a TissueLyser, and DNA samples were stored at $-20^{\circ} \mathrm{C}$.

\section{Bulked segregant analysis}

BSA was used in conjunction with SSR analysis (Michelmore et al., 1991) to find markers linked to RWC genes. Tolerant and sensitive bulks were prepared from $\mathrm{F}_{2}$ individuals by pooling aliquots containing equivalent amounts of total DNA, approximately $50 \mathrm{ng} / \mu \mathrm{L}$ each from 10 sensitive and 10 tolerant $\mathrm{F}_{2}$ plants selected based on phenotypic assessments. SSR primers were then screened using the parents and the 2 bulk DNA samples, from which some primer combinations revealed bands that were polymorphic not only among parental genotypes but also between the bulk DNA pair. Based on the evaluations of the DNA bulks, individual $F_{2}$ plants were analyzed with cosegregating primers to confirm SSR marker linkage to the RWC. PCRs were performed as described by Röder et al. (1998) in a Biometra thermal cycler (Germany) with the following protocol: 3 min at $94^{\circ} \mathrm{C} ; 35$ cycles of $30 \mathrm{~s}$ at $94^{\circ} \mathrm{C}, 30 \mathrm{~s}$ for annealing $\left(55^{\circ}-60^{\circ} \mathrm{C}\right)$, and 30 $\mathrm{s}$ at $72^{\circ} \mathrm{C}$, and a final extension of $10 \mathrm{~min}$ at $72^{\circ} \mathrm{C}$. PCR products were separated on $3 \%$ agarose gel and electrophoresed at $180 \mathrm{~V}$ for $80 \mathrm{~min}$. Gels were stained with ethidium bromide, and visualized and photographed under UV light. The AntMap version 1.2 software was employed to construct the linkage map, and QTL cartographer was used to detect QTLs for the RWC.

\section{Data analysis}

Single-marker analysis to detect the main effect of the QTLs was performed using the method of Liu (1998). A significant association between a tested marker and a QTL for RWC was detected by primary ANOVA; linear regression estimated the contribution of a markerlinked QTL to total variation in the RWC in $\mathrm{F}_{2}$ lines where a QTL was claimed. All statistical procedures were performed in Excel and SAS (2001). 


\section{RESULTS AND DISCUSSION}

A total of 41 SSR primer pairs were used to screen the parents; 5 polymorphic primer pairs were identified for polymorphisms between parents, and 3 of them amplified polymorphic bands strongly among the $\mathrm{F}_{2}$ population. The continuous distribution of the RWC in the $\mathrm{F}_{2}$ population ranged from 62 to $86 \%$. In this study, frequency distributions were examined to detect the extent of phenotypic variation occurring with high and low RWC. As expected, distribution was normal, proving that RWC is affected not by a single gene but by multiple genes (Figure 1). The linkage relationship between the SSR markers (Xgwm182, Xgwm292, and Xgwm410) and the RWC as an indicator for drought tolerance genes was estimated using an $F_{2}$ population derived from S78-11 x Tajan. The SSR primers GWM182, GWM292, and GWM410 amplified fragments at 240, 180, and $300 \mathrm{bp}$, respectively (Figure 2). Typically, for GWM182, 5 of 10 individuals possessed tolerant bulk profiles (Figure 2). Some studies have reported using BSA to find links for specific genes (Michelmore et al., 1991; Govindaraj et al., 2005). One-way ANOVA was carried out using marker genotypes as groups.

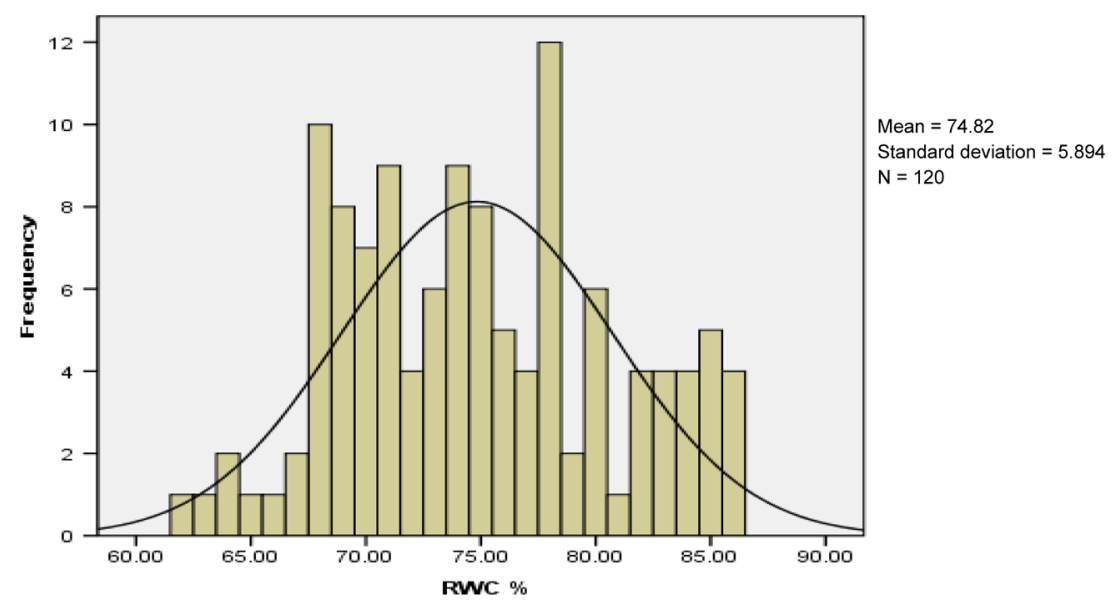

Figure 1. Frequency distribution of the relative water content in the field experiment in the $\mathrm{F}_{2}$ population.

ANOVA on the GWM182, GWM292, and GWM410 marker genotypes for RWC established a significant association between these 3 markers and the phenotype (Table 1). The genetic distance between the SSR markers (Xgwm182, Xgwm292, and Xgwm410) and drought tolerance genes were determined as $28.97,32.02$, and $30.41 \mathrm{cM}$, respectively, with logarithm (base 10) of odds, scores of 4.76, 3.60, and 4.24, respectively (Table 2). Therefore, the SSR markers were linked to the QTLs for RWC. The potential value of RWC for breeding under drought stress conditions has been demonstrated by Schonfeld et al. (1988) in winter bread wheat.

These authors also showed that RWC is inherited quantitatively and controlled by genes with additive effects, and a positive correlation between grain yield and RWC has been observed in durum wheat (Merah, 2001), bread wheat (Schonfeld et al., 1988; Tahara et al., 1990; Singh and Patel, 1996), and oat (Peltonen-Sainio and Mäkelä, 1995). 

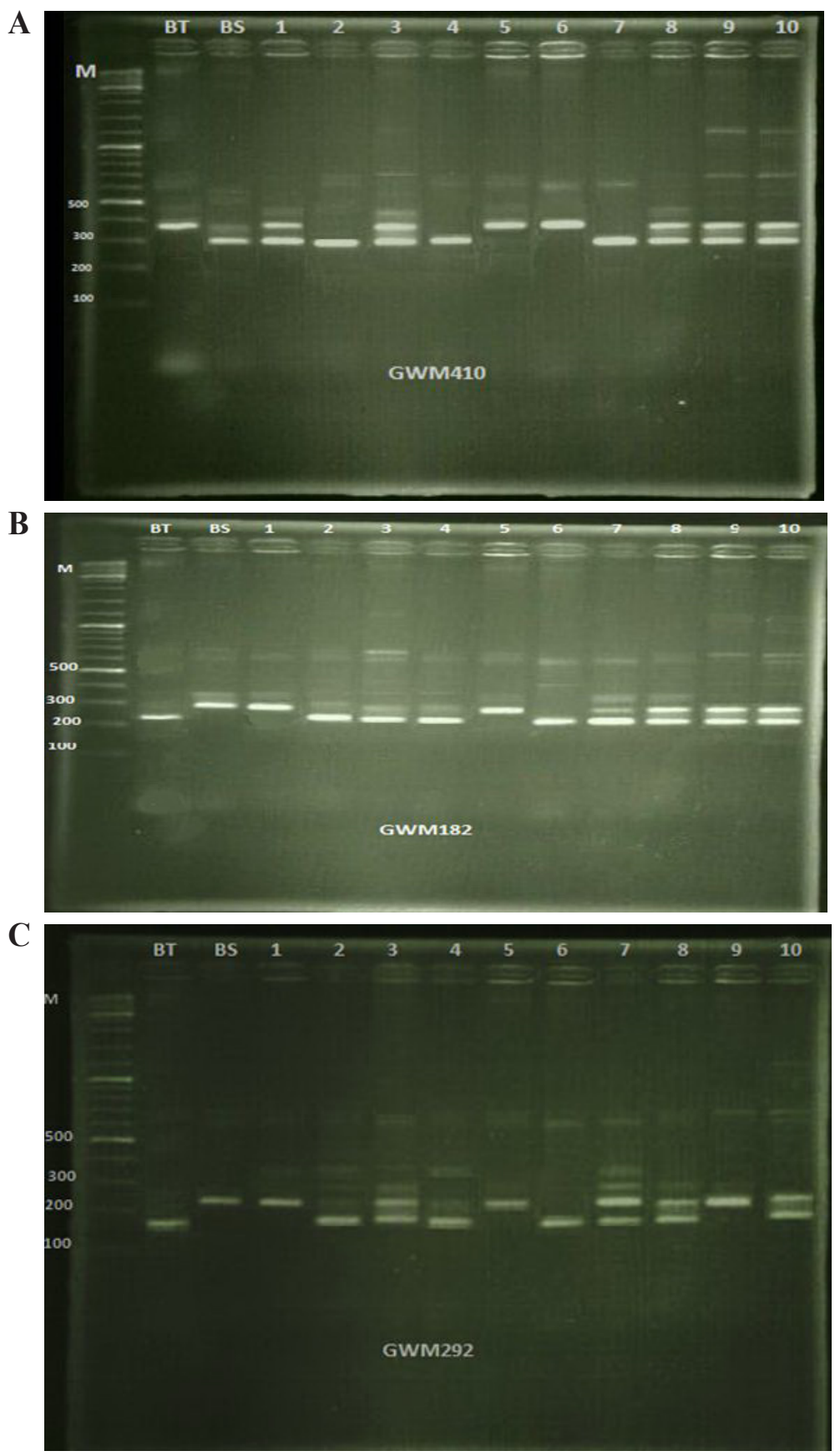

Figure 2. Marker banding patterns in the $\mathrm{F}_{2}$ population of wheat derived from S-78-11 x Tajan for SSR markers GWM410 (A), GWM182 (B) and GWM292 (C) linked to relative water content running on 3\% agarose gel stained with ethidium bromide, 10 samples plus two tolerant bulk (BT) and sensitive bulk (BS). Lane $M=100$-bp ladder. Lanes 1 to $10=$ progenies. 


\begin{tabular}{|c|c|c|c|c|}
\hline Marker & Source & d.f. & SS & MS \\
\hline \multirow[t]{3}{*}{ GWM182 } & Between groups & 2 & 1258.47 & $629.23 * *$ \\
\hline & Within groups & 117 & 2822.82 & 24.12 \\
\hline & Total & 119 & 4081.3 & \\
\hline \multirow[t]{3}{*}{ GWM292 } & Between groups & 2 & 907.91 & $453.95 * *$ \\
\hline & Within groups & 117 & 3173.38 & 27.12 \\
\hline & Total & 119 & 4081.3 & \\
\hline \multirow[t]{3}{*}{ GWM410 } & Between groups & 2 & 865.95 & $432.97 * *$ \\
\hline & Within groups & 117 & 3215.34 & 27.48 \\
\hline & Total & 119 & 4081.3 & \\
\hline
\end{tabular}

**Significant at $1 \%$. d.f. $=$ degrees of freedom; $\mathrm{SS}=$ sum of squares; $\mathrm{MS}=$ mean squares.

Table 2. Specification of quantitative trait loci (QTL) related to the relative water content in the $120 \mathrm{~F}_{2}$ plants of
S-78-11 x Tajan.
\begin{tabular}{lcccc}
\hline Marker & QTL $(\mathrm{cM})$ & Chromosome & LOD & $\mathrm{R}^{2}(\%)$ \\
\hline GWM182 & 28.97 & $5 \mathrm{D}$ & 4.76 & 30 \\
GWM292 & 32.02 & 5D & 3.60 & 22 \\
GWM410 & 30.41 & $5 \mathrm{~A}$ & 4.26 & 21 \\
\hline
\end{tabular}

$\mathrm{LOD}=$ logarithm of the odds.

The 3 polymorphic markers identified based on the analysis of bulked extremes were used to screen $120 \mathrm{~F}_{2}$ individuals to establish the association between the respective markers and phenotypes. The single-marker analysis revealed that GWM182, GWM292, and GWM410 were linked to QTLs and accounted for 30, 22, and $21 \%$ of the total variation in RWC in the $\mathrm{F}_{2}$ population, respectively. The 3 co-dominant microsatellite markers could determine the heterozygotes and would serve as an important tool to transfer the RWC genes into other wheat cultivars.

\section{CONCLUSIONS}

A population consisting of $120 \mathrm{~F}_{2}$ individuals from a cross between the drought-tolerant S-78-11 and drought-sensitive Tajan cultivars were analyzed for their segregation pattern of the difference of RWC under drought stress conditions. Multiple genes control drought tolerance in wheat.

The SSR markers GWM182 and GWM292 on chromosome 5D and GWM410 on chromosome $5 \mathrm{~A}$ exhibited a significant association with drought tolerance, respectively, accounting for 30,22 , and $21 \%$ of the total variation, respectively. The 3 genetic loci, especially GWM182 on chromosome 5D, can be used in marker-assisted selection methods for drought tolerance breeding in wheat.

\section{ACKNOWLEDGMENTS}

The authors extend special thanks to Dr. Mihdzar Abdul Kadir (University Putra Malaysia) for his contribution in QTL analysis.

\section{REFERENCES}

Barrs HD and Watherley PE (1962). A re-examination of the relative turgidity technique for estimating water deficit in 
leaves. Aust. J. Biol. Sci. 15: 413-428.

Blum A (1989). Osmotic adjustment and growth of barley genotypes under drought stress. Crop Sci. 29: 230-233.

Farquhar GD and Richards RA (1984). Isotopic composition of plant carbon correlates with water-use efficiency of wheat genotypes. Aust. J. Plant Physiol. 11: 539-552.

Govindaraj P, Arumugachamy S and Maheswaran M (2005). Bulked segregant analysis to detect main effect QTL associated with grain quality parameters in Basmati 370/ASD 16 cross in rice (Oryza sativa L.) using SSR markers. Euphytica 144: 61-68.

Liu BH (1998). Statistical Genomics. CRC Press, Boca Raton, 387-416.

Merah O (2001). Potential importance of water status traits for durum wheat improvement under Mediterranean conditions. J. Agric. Sci. 137: 139-145.

Michelmore RW, Paran I and Kesseli RV (1991). Identification of markers linked to disease-resistance genes by bulked segregant analysis: a rapid method to detect markers in specific genomic regions by using segregating populations. Proc. Natl. Acad. Sci. U. S. A. 88: 9828-9832.

Peltonen-Sainio P and Mäkelä P (1995). Comparison of physiological methods to assess drought tolerance in oats. Acta Agric. Scand. 45: 32-38.

Price A and Courtois B (1999). Mapping QTLs associated with drought resistance in rice: progress, problems and prospects. Plant Growth Reg. 29: 123-133.

Röder MS, Korzun V, Wendehake K, Plaschke J, et al. (1998). A microsatellite map of wheat. Genetics 149: 2007-2023.

SAS Statistical Analysis Software (2001). Version 9.1. SAS Institute, Cary.

Schonfeld MA, Johnson RC, Carver BF and Mornhinweg DW (1988). Water relations in winter wheat as drought resistance indicators. Crop Sci. 28: 526-531.

Sinclair TR and Ludlow MM (1985). Who taught plants thermodynamics? The unfulfilled potential of plant water potential. Aust. J. Plant Physiol. 12: 213-217.

Singh J and Patel AL (1996). Water-status, gas exchange, proline accumulation and yield of wheat in response to water deficit. Ann. Biol. 12: 77-81.

Tahara M, Carver BF, Johnson RC and Smith EL (1990). Relationship between relative water content during reproductive development and winter wheat grain yield. Euphytica 49: 255-262.

Tanksley SD (1993). Mapping polygenes. Ann. Rev. Genet. 27: 205-233.

Teulat B, This D, Khairallah M, Borries C, et al. (1998). Several QTLs involved in osmotic-adjustment trait variation in barley (Hordeum vulgare L.). Theor. Appl. Genet. 96: 688-698.

Tuberosa R and Salvi S (2006). Genomics-based approaches to improve drought tolerance of crops. Trends Plant Sci. 11: 405-412. 